\title{
Effect of different semimonolithic designs on fracture resistance and fracture mode of translucent and high-translucent zirconia crowns
}

This article was published in the following Dove Press journal: Clinical, Cosmetic and Investigational Dentistry

\author{
Fahad Bakitian ${ }^{1,2}$ \\ Przemek Seweryniak ${ }^{3}$ \\ Evaggelia Papia' \\ Christel Larsson' \\ Per Vult von Steyern'
}

'Department of Materials Science and Technology, Faculty of Odontology, Malmö University, Malmö, Sweden; 2Department of Restorative Dentistry, Faculty of Dentistry, Umm Al-Qura University, Makkah, Saudi Arabia; ${ }^{3}$ Commercial Dental Laboratory, Malmö, Sweden
Correspondence: Fahad Bakitian Department of Materials Science and Technology, Faculty of Odontology, Malmö University, Carl Gustafs väg 34, 20506 Malmö, Sweden

Tel +46406658583

Fax +46 406657075

Email fahad.bakitian@mau.se
Purpose: The aim of this study was to describe different designs of semimonolithic crowns made of translucent and high-translucent zirconia materials and to evaluate the effect on fracture resistance and fracture mode.

Methods: One hundred crowns with different designs were produced and divided into five groups ( $\mathrm{n}=20$ ): monolithic (M), partially veneered monolithic (semimonolithic) with $0.3 \mathrm{~mm}$ buccal veneer (SM0.3), semimonolithic with $0.5 \mathrm{~mm}$ buccal veneer (SM0.5), semimonolithic with $0.5 \mathrm{~mm}$ buccal veneer supported by wave design (SMW), and semimonolithic with $0.5 \mathrm{~mm}$ buccal veneer supported by occlusal cap design (SMC). Each group was divided into two subgroups $(\mathrm{n}=10)$ according to the materials used, translucent and high-translucent zirconia. All crowns underwent artificial aging before loading until fracture. Fracture mode analysis was performed. Fracture loads and fracture modes were analyzed using two-way ANOVA and Fisher's exact probability tests $(P \leq 0.05)$.

Results: SM0.3 design showed highest fracture loads with no significant difference compared to $\mathrm{M}$ and SMW designs $(P>0.05)$. SM0.5 design showed lower fracture loads compared to SMW and SWC designs. Crowns made of translucent zirconia showed higher fracture loads compared to those made of high-translucent zirconia. M, SM0.3, and all but one of the SMC crowns showed complete fractures with significant differences in fracture mode compared to SMW and SM0.5 crowns with cohesive veneer fractures $(P \leq 0.05)$.

Conclusion: Translucent and high-translucent zirconia crowns might be used in combination with $0.3 \mathrm{~mm}$ microcoating porcelain layer with semimonolithic design to enhance the esthetic properties of restorations without significantly decreasing fracture resistance of the crowns. If $0.5 \mathrm{~mm}$ porcelain layer is needed for a semimonolithic crown, wave design or cap design might be used to increase fracture resistance. In both cases, fracture resistance gained is likely to be clinically sufficient as the registered fracture loads were high in relation to expected loads under clinical use.

Keywords: monolithic zirconia, porcelain veneer, Y-TZP

\section{Introduction}

Yttrium oxide-stabilized tetragonal zirconia polycrystal material (Y-TZP) has become accepted over the past decades as a biocompatible restorative material, ${ }^{1}$ often considered to be the material of choice for many conditions in prosthetic dentistry. ${ }^{2}$ Generally, all ceramic systems based on Y-TZP can be found in the following two major designs: traditional bi-layered design and a more recent monolithic design.

Bi-layered Y-TZP design is relatively well documented with respect to both clinical follow-up studies and laboratory studies. ${ }^{3-6}$ However, several clinical studies have 
shown that despite the excellent mechanical properties of Y-TZP, superficial chip-off fracture in the veneering ceramic is a common clinical problem for this type of design. ${ }^{3-5}$ The reason for veneering Y-TZP restorations, in the traditional design, is that the optical properties of the material itself are poor, ${ }^{2,7}$ and the material needs to be veneered with a highly esthetic ceramic material to achieve an acceptable resemblance to natural tooth structure. Low-strength vitreous ceramics, such as porcelain, are then fused to the high strength Y-TZP core material to combine the strength with better esthetic outcomes. ${ }^{7}$

Monolithic design, on the other hand, has been increasingly popular over the last decade since it is believed to withstand high loads during function without the risk of chip-off fractures of veneering materials. To overcome the poor optical properties of the traditional Y-TZP material, translucent zirconia materials were developed for monolithic restorations, ${ }^{8}$ often referred to as monolithic translucent zirconia. The first generation of translucent zirconia materials had mechanical properties comparable to the traditional Y-TZP with promising laboratory and short-term clinical studies. ${ }^{9-12}$

Recently, there were notable attempts for making hightranslucent monolithic zirconia to be used in highly esthetic clinical cases. Nevertheless, the translucency that can be gained without tampering with the strength of the material is very limited, and it is not possible to gain translucency above a certain level without losing the mechanical properties of the material to some extent. ${ }^{8,13,14}$ This new generation of high-translucent zirconia materials with scarce clinical data might be considered to be used with caution due to their low mechanical properties and doubtful aging stability. ${ }^{8,13,14}$ Therefore, it would be desirable to maintain the strength of the translucent zirconia materials and still gain better esthetic outcomes without the risks that are associated with full veneering or the increased translucency of high-translucent zirconia.

Partially veneered monolithic (semimonolithic) crown design with porcelain coverage on the buccal area only might be a feasible method to overcome both the issues with chip-off fractures and the poor esthetics of full monolithic zirconia crowns. In this way, it might be possible to exclude the porcelain on surfaces subjected to wear and high loads such as the occlusal tables and the marginal ridges. Such a design has become common today but is not always sanctioned in the manufacturers' recommendations for the materials and is consequently used without the support from manufacturers' instructions. ${ }^{15,16}$ Scientific studies on this subject are lacking.
Different alternative semimonolithic crown designs are, therefore, proposed and investigated in this study. They can be described as microcoating design, reduced veneer design, wave support design, and occlusal cap-like projection design; all are suggested to enhance the esthetics of the restoration without decreasing the overall strength. It has not yet been evaluated, however, which of the proposed designs that are to be recommended, or if the designs add strength to the crowns compared to monolithic ones.

Hence, the aim of the present study was to describe different semimonolithic crown designs for translucent and hightranslucent zirconia materials and to evaluate their fracture resistance and fracture mode under the null hypothesis that all designs for both materials included would perform equally.

\section{Methods \\ Study design}

A total of 100 standardized crowns with different designs were produced and divided into five groups $(n=20)$ (Figure 1): monolithic (M), partially veneered monolithic (semimonolithic) with $0.3 \mathrm{~mm}$ microcoated buccal veneer (SM0.3), semimonolithic with $0.5 \mathrm{~mm}$ buccal veneer (SM0.5), semimonolithic with $0.5 \mathrm{~mm}$ buccal veneer supported by wave design (SMW), and semimonolithic with $0.5 \mathrm{~mm}$ buccal veneer supported by occlusal cap design (SMC).

Each group was divided into two subgroups $(n=10)$ according to the crown materials used, translucent zirconia (BruxZir $^{\circledR}$ Solid Zirconia; Glidewell Dental Laboratories, Newport Beach, CA, USA) and high-translucent zirconia (Zirkon BioStar HT; Siladent, Goslar, Germany).

\section{Production of crowns and abutment dies}

A metal master die in the shape of a prepared molar, designed for an all-ceramic crown, was replicated by using a silicone impression material (President ${ }^{\circledR}$; Coltene AG, Altstätten, Switzerland). The impression was poured with die stone (Vel-Mix; Kerr Corporation, Orange, CA, USA) to produce a master die. A wax-up of a full anatomical crown was fabricated on the master die. A double scan technique was then performed with a laboratory scanner (D900L; 3Shape, Copenhagen, Denmark) to create CAD files for both the wax-up crown and the master die. The semimonolithic crowns were designed with specific dimensions through the CAD software (Figure 1). The CAD data were sent to a certified milling center (Cosmodent AB, Malmö, Sweden) for the production of 100 zirconia crowns with different designs (Figure 2). In the same production step, 100 abutment dies 

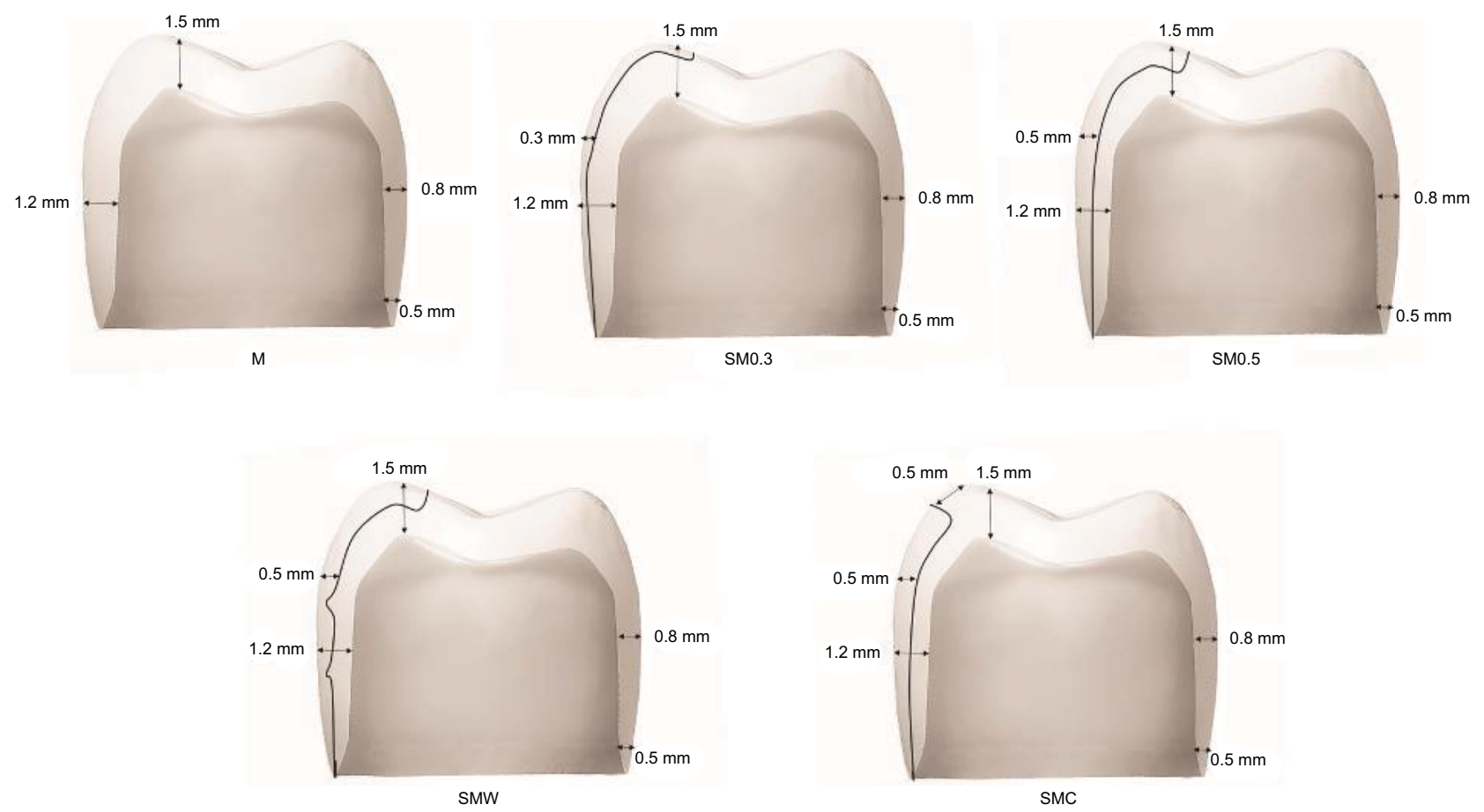

Figure I Illustrations showing dimensions of the crown designs.

Notes: The black line represents the interface between the core and the veneer. The arrows denote the local dimensions of the crowns. M, monolithic; SM0.3, semimonolithic with $0.3 \mathrm{~mm}$ buccal veneer; SM0.5, semimonolithic with $0.5 \mathrm{~mm}$ buccal veneer; SMW, semimonolithic with $0.5 \mathrm{~mm}$ buccal veneer supported by wave design; SMC, semimonolithic with $0.5 \mathrm{~mm}$ buccal veneer supported by occlusal cap design.

were milled from a polymer material (Polyoxymethylene; Nordbergs Tekniska AB, Stockholm, Sweden).

The crowns were veneered buccally with a compatible porcelain recommended by the manufacturer (IPS e.max Ceram; Ivoclar Vivadent, Schaan, Liechtenstein). A specially made knife was used to standardize the dimensions of the crowns in the different groups using a method that is used in previous studies. ${ }^{17,18}$ The porcelain was applied using specific brushes in several layers (liner, wash, dentin 1, dentin 2, glaze) according to the manufacturer's recommendations. While applying the porcelain layers, the crown over the abutment was placed and fixated to the brass holder that was rotated manually, permitting the knife to carve the porcelain into the final shape (Figure 3). Each layer was separately fired in a calibrated furnace (Ivoclar P 500; Ivoclar Vivadent) at specific temperatures, again according to the manufacturer's recommendations.

\section{Artificial aging and cementation}

All crowns underwent three steps of artificial aging: thermocycling, storing in a $37^{\circ} \mathrm{C}$ moist environment and cyclic preload. The crowns were first subjected to 10,000 thermocycles in a specially built thermocycling device containing two water baths at different temperatures, 5 and $55^{\circ} \mathrm{C}$. Each cycle lasted for 60 seconds, 20 seconds in each bath, and 10 seconds for transfer between the baths. In the present study, the crowns were subjected to thermocycling before cementation to prevent partly loose crowns during the preload and load to fracture tests since previous studies have shown the deleterious impact of the laboratory thermocycling on the integrity of bonding between the crown and cement. ${ }^{19}$

Before cementation, the abutment dies were air-abraded with $110 \mu \mathrm{m}$ aluminum oxide at a pressure of 2 bar for $15 \mathrm{sec}-$ onds, at a distance of $10 \mathrm{~mm}$ perpendicular to the die surface to increase the surface area. The inner surfaces of the crowns were sandblasted using $50 \mu \mathrm{m}$ aluminum oxide at a pressure of $2 \mathrm{bar}$, and then, they were rinsed with water according to the manufacturer's recommendations as was suggested in a previous study by Shahin and Kern. ${ }^{20}$ After that, the crowns were cemented to the abutment dies with a compatible resin cement according to the manufacturers' recommendation (Panavia F 2.0; Kuraray Medical Inc, Okayama, Japan). During the cement setting, all crowns were loaded in the direction of insertion with a load of $15 \mathrm{~N}$ for a period of 60 seconds.

After cementation, the specimens were stored in a plastic container inside an incubator (Memmert Incubator; Memmert, $\mathrm{GmbH}$, Schwabach, Germany) at a temperature of $37^{\circ} \mathrm{C}$ for 60 days. All specimens were wrapped in a wet paper to 
A

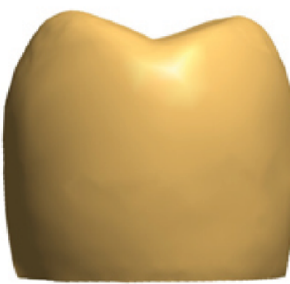

M

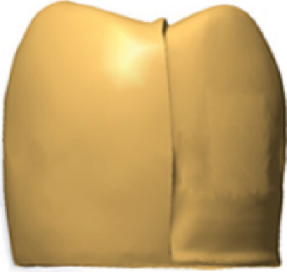

SM0.3

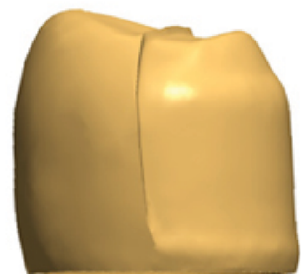

SM0.5

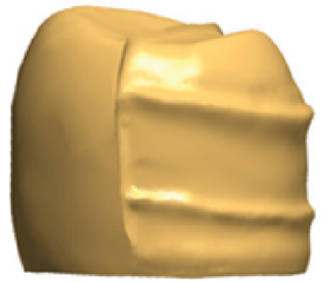

SMW

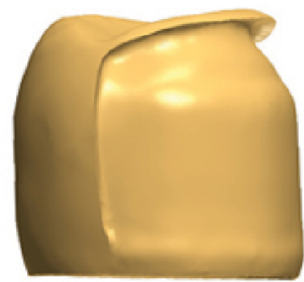

SMC

B

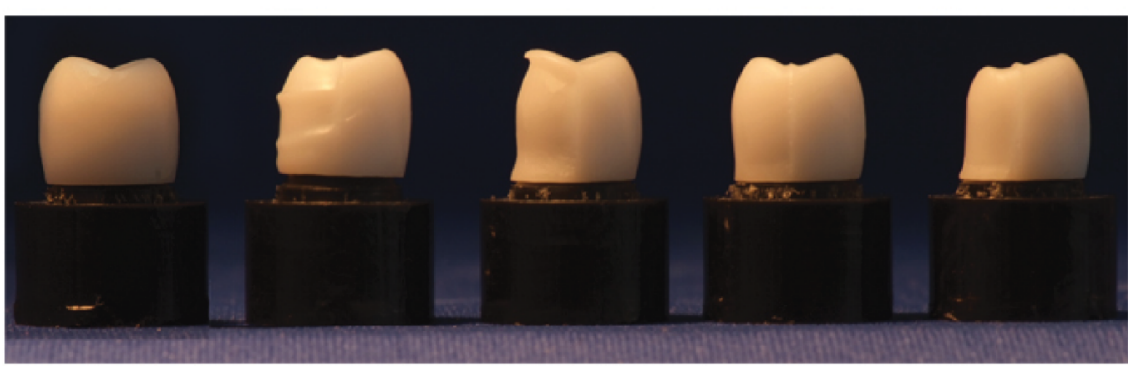

Figure 2 Different crown designs shown: $(\mathbf{A})$ in the $C A D$ software and $(\mathbf{B})$ after milling and sintering.

Notes: M, monolithic; SM0.3, semimonolithic with $0.3 \mathrm{~mm}$ buccal veneer; SM0.5, semimonolithic with $0.5 \mathrm{~mm}$ buccal veneer; SMW, semimonolithic with $0.5 \mathrm{~mm}$ buccal veneer supported by wave design; SMC, semimonolithic with $0.5 \mathrm{~mm}$ buccal veneer supported by occlusal cap design.

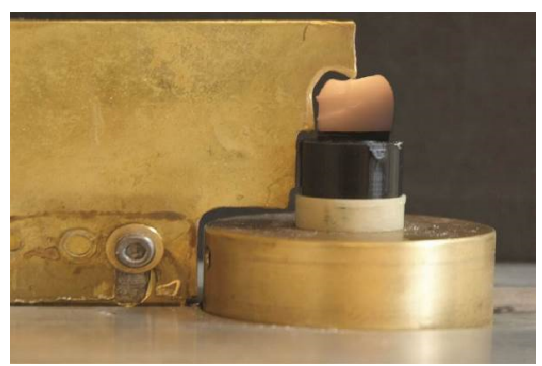

Figure 3 Porcelain build-up step.

Note: The crown with abutment placed on the brass holder, and the specially made knife was used to standardize dimensions of semimonolithic crowns.

create a moist environment like the oral cavity and to prevent desiccation of the luting cement.

In the final stage of artificial aging, the specimens underwent 10,000 cycles of preload at $30-300 \mathrm{~N}$ in the form of a sine wave of $1 \mathrm{~Hz}$. The specimens were mounted submerged in water at $10^{\circ}$ inclination relative to the vertical plane in preloading machine (MTI Engineering AB, Lund, Sweden/Pamaco AB, Malmö, Sweden) as was suggested in previous laboratory studies. ${ }^{17,18}$ The load was applied at the highest point of the inner buccal cusp inclination by a stainless steel ball with a diameter of $4 \mathrm{~mm}$ (Figure 4). A 0.2-mm thick plastic foil (PE-Baufolie, Probau; Bauhaus, Zug, Switzerland) was placed between the ball and the crown
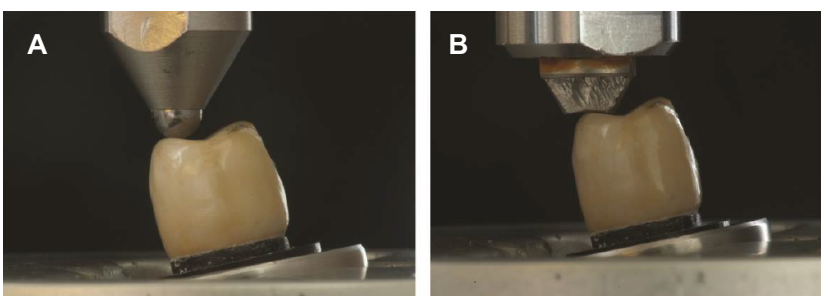

Figure 4 Test set-ups used for: (A) cyclic preload and (B) load to fracture.

Note: During the test, the crowns were submerged in water, and a plastic foil was used to spread the load evenly over loaded surfaces. 
during preloading to distribute the loading forces evenly as was suggested by Kelly. ${ }^{21}$

In the present study, artificial aging was used to mimic the aging procedure in the clinical situation to evaluate its effect on the fracture resistance of the crowns. There is no consensus about what constitutes the optimal artificial aging procedure in respect of the number of cycles, temperature range, or storage time. ${ }^{22,23}$ In this study, thermocycling and preload tests were adopted from previous studies conducted by the same research group to enable comparisons. ${ }^{24,25}$

\section{Load to fracture}

After artificial aging, all specimens were loaded to fracture by using a universal testing machine (Instron 4465; Instron Co. Ltd, Norwood, MA, USA). The same test setup as during cyclic preload was used for this step, but the load was applied at the highest point of the inner buccal cusp inclination of the crown, with a special indenter designed to accommodate this inclination to prevent sliding movement at load (Figure 4). The crosshead speed was $0.255 \mathrm{~mm} / \mathrm{min}$. Load at fracture was registered, and the fracture was defined as an apparent crack in the crown, a load drop, or an acoustic event, whichever came first. The acoustic event was ascertained by hearing a cracking sound that is convoyed by the changes in the load-path graph on a computer that is connected to the testing machine.

\section{Analysis of fracture mode}

The fracture surfaces of the crowns were assessed by one examiner. A gross visual and microscopic assessments (Wild M3; Wild Heerbrugg, Heerbrugg, Switzerland) were performed to classify the fracture mode into: adhesive (denoting fracture in the interface between the veneer and the core), cohesive (denoting fracture within the buccal veneer, ie, chipping), or complete (denoting the fracture of both the veneer and the core).

\section{Statistical analysis}

The differences in fracture load data among the tested groups were analyzed using a two-way ANOVA, followed by Tukey's post hoc test (IBM SPSS Statistics 22; SPSS Inc., Chicago, IL, USA). Fracture mode was analyzed using Fisher's exact probability test. Results were considered statistically significant at $5 \%$ level of significance $(P \leq 0.05)$.

\section{Results \\ Fracture load}

There were significant differences in fracture load among the groups depending on the materials used and the different designs tested $(P \leq 0.05)$ (Figure 5). Generally, crowns made of translucent zirconia showed higher fracture loads compared to those made of high-translucent zirconia, regardless of design $(P<0.001)$. Regarding design, the semimonolithic groups with $0.3 \mathrm{~mm}$ microcoated buccal veneer (SM0.3) showed the highest fracture loads of all the other semimonolithic groups, with no significant difference compared to the $\mathrm{M}$ and SMW groups $(P>0.05)$. The crowns in the SM0.5 group showed significantly lower fracture loads compared to the crowns in the SMW and SMC groups. By comparing the designs in both materials used: the M, SM0.3, and SMW groups of translucent zirconia showed significantly higher fracture loads compared to all the groups of high-translucent zirconia. There was no significant difference between the $\mathrm{M}$ group of high-translucent zirconia compared to the SMC and SM0.5 groups of translucent zirconia.

\section{Fracture mode}

There were substantial differences among the groups, based on the designs, in the way that the crowns fractured. Two fracture modes were seen visually and under the light microscope: complete fracture and cohesive veneer fracture (Figure 6). All crowns in the M and SM0.3 groups (100\%), and all but one of the crowns in the SMC groups $(95 \%)$ showed complete fractures with significant differences $(P \leq 0.05)$ in the fracture mode compared to the crowns in the other two groups (SMW and SM0.5). The SMW and SM0.5 groups showed (70 and $55 \%$, respectively) cohesive veneer fractures (Figure 7). None of the tested crowns showed an adhesive fracture.

\section{Discussion}

The null hypothesis assuming that crowns with different semimonolithic designs of both translucent and high-translucent zirconia materials would have equal fracture loads was rejected. The results showed that the semimonolithic crowns with a microcoating porcelain layer withstood the highest fracture loads among the semimonolithic crowns tested, close to the loads seen in the monolithic crowns with no statistical differences in these two crown designs. The results in the groups with semimonolithic wave design showed fracture loads at approximately the same level of the groups with the semimonolithic microcoating design. The fracture loads of the semimonolithic crowns veneered with porcelain $0.5 \mathrm{~mm}$ thick were, however, significantly lower compared to the other designs studied. This might imply that a thicker porcelain layer results in lower strength values, reaching those seen with traditionally veneered Y-TZP crowns where the veneered porcelain layer is usually within the $1-1.5 \mathrm{~mm}$ 


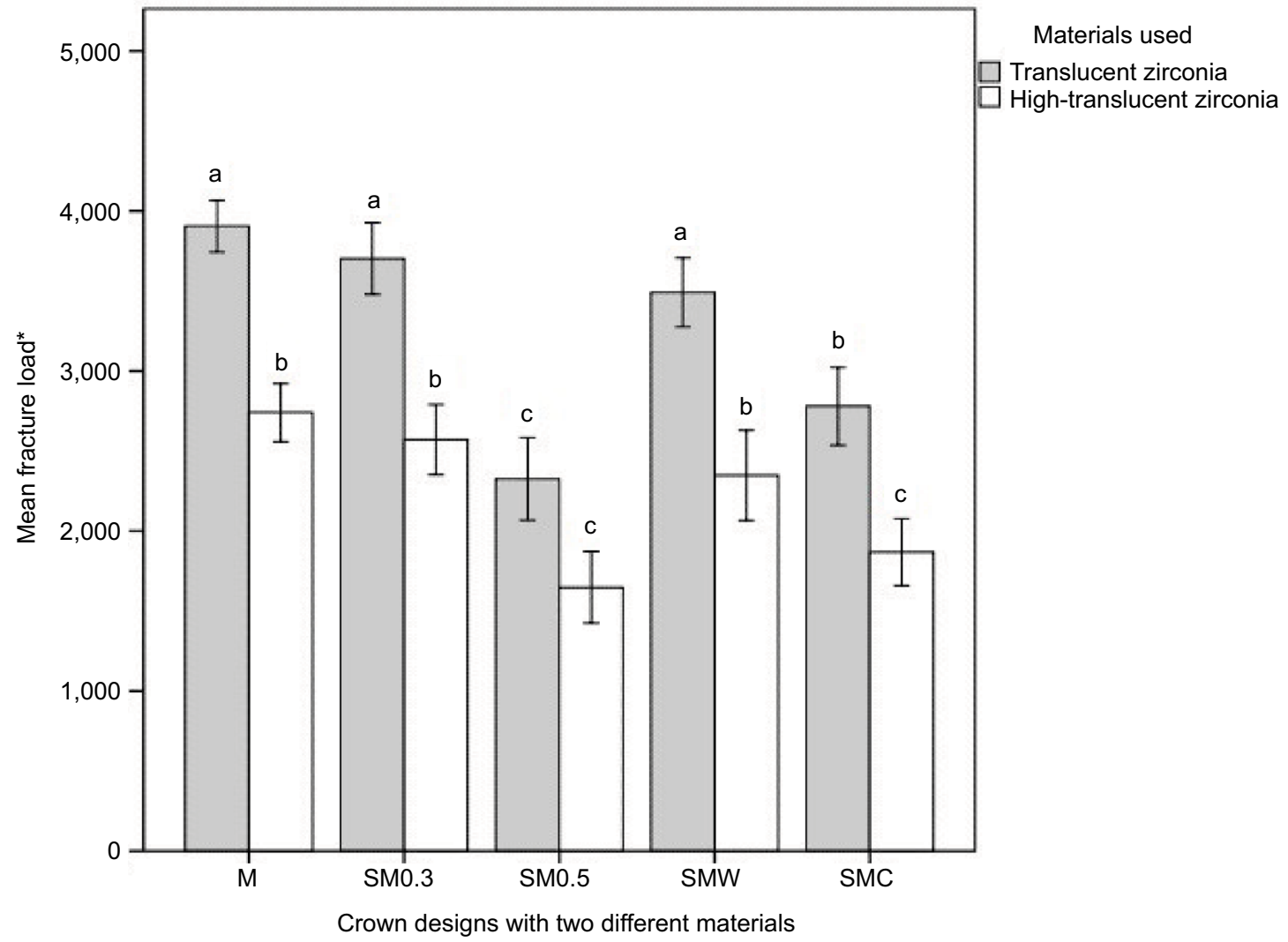

Figure 5 Comparison of mean fracture load (Newton) among the crown designs with two different materials.

Notes: *Mean with the same letters (denoted a, b, and c) did not show any significant difference in fracture load ( $P>0.05$ ). M, monolithic; SM0.3, semimonolithic with a $0.3 \mathrm{~mm}$ buccal veneer; SM0.5, semimonolithic with a $0.5 \mathrm{~mm}$ buccal veneer; SMW, semimonolithic with a 0.5 mm buccal veneer supported by wave design; SMC, semimonolithic with a $0.5 \mathrm{~mm}$ buccal veneer supported by occlusal cap design.
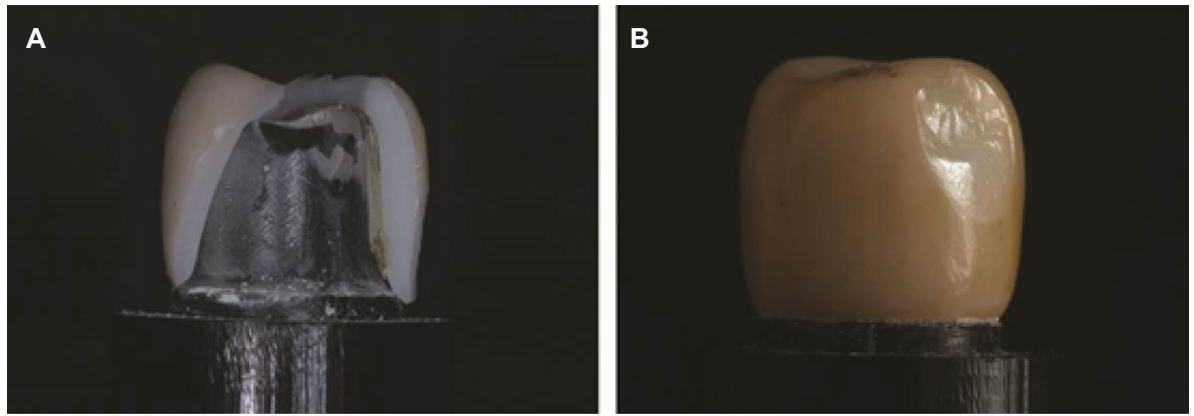

Figure 6 Fracture modes of the crowns: (A) complete fracture in M, SM0.3, and SMC groups and (B) cohesive veneer fracture in SMW and SM0.5 groups. Notes: M, monolithic; SM0.3, semimonolithic with $0.3 \mathrm{~mm}$ buccal veneer; SM0.5, semimonolithic with $0.5 \mathrm{~mm}$ buccal veneer; SMW, semimonolithic with $0.5 \mathrm{~mm}$ buccal veneer supported by wave design; SMC, semimonolithic with $0.5 \mathrm{~mm}$ buccal veneer supported by occlusal cap design.

range in thickness. ${ }^{8,13}$ This assumption, however, is not confirmed in the present study since fully veneered crowns were not included. One postulate in the study design was that the overall thickness of the crowns should be identical in all the groups, simulating a standardized preparation depth regardless of the design to be tested. This means that the thicker the veneering porcelain, the thinner the supporting core material underneath became, which probably played a significant role in the results of the study. This is worth considering when analyzing the results. It is always a limiting factor in the clinical situation where preparation that is too aggressive removes important, often healthy, tooth structure, thus affecting the prognosis for the tooth vitality. ${ }^{26}$ Hence, one indication for using monolithic zirconia is that it enables tooth tissue to 


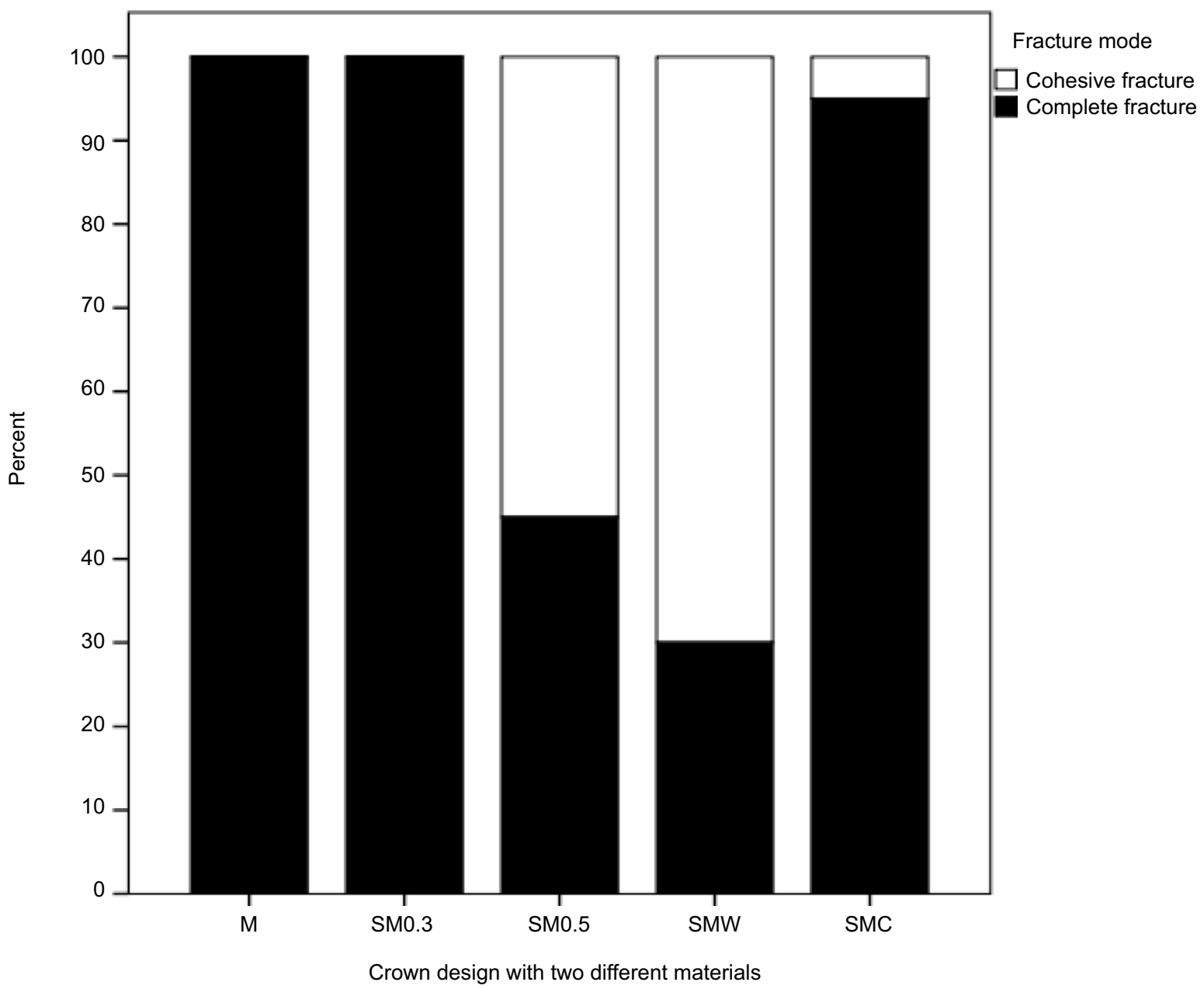

Figure 7 Comparison of fracture mode among the crown designs with two different materials.

Notes: M, monolithic; SM0.3, semimonolithic with a $0.3 \mathrm{~mm}$ buccal veneer; SM0.5, semimonolithic with a $0.5 \mathrm{~mm}$ buccal veneer; SMW, semimonolithic with a $0.5 \mathrm{~mm}$ buccal veneer supported by wave design; SMC, semimonolithic with a $0.5 \mathrm{~mm}$ buccal veneer supported by occlusal cap design.

be saved due to a material strength that makes a restoration durable even when it is designed as a thin $(0.5 \mathrm{~mm})$ crown coping. ${ }^{10}$ Thus, it might not be considered clinically relevant to test crown specimens where the overall thickness increases by increasing the thickness of the veneer portion since this would have necessitated a different preparation depth for the different groups in the study. It was, therefore, decided that the same clinical situation with respect to the preparation depth should be represented in all groups. Consequently, increasing the porcelain thickness resulted in a thinner core, providing less support for the $0.5 \mathrm{~mm}$ porcelain layer than for the crowns with a microcoating layer. Furthermore, the larger the volume of veneering porcelain, the greater the probability of flaw populations with a critical flaw existing in the material portions under stress during loading. ${ }^{27,28}$ This might be another reason for the lower fracture loads in the groups with $0.5 \mathrm{~mm}$ veneer thickness compared to the groups with $0.3 \mathrm{~mm}$ veneer thickness.

Previous studies have suggested the minimum fracture strength for a dental restoration, allowing it to be used in all areas of the mouth. ${ }^{29,30}$ These suggestions were proposed in line with the data relating to average maximum bite forces, which vary in different areas in the mouth and range from 90 to 900 N. ${ }^{29,30}$ Maximum bite forces differ from patient to patient, and extreme loads have been registered in some isolated individuals. The highest measured load known to the authors was 4,000 $\mathrm{N}$ measured in a single individual, whereas the same source reported 2,000 $\mathrm{N}$ as the second highest load measured in another single person. ${ }^{30}$ It is not reasonable, however, to aim for such high bite forces when designing restorations since the vast majority of patients seldom reach or exceed $1,000 \mathrm{~N}$, and normal chewing forces are significantly 
lower. With that in mind, all the groups in the present study performed well and with a high margin of safety in respect of the expected load under function in the oral environment.

Some cases require the use of thicker porcelain layers, $\geq 0.5 \mathrm{~mm}$, for a better esthetic outcome. In such situations, it might be advantageous to protect the veneer by using other means of core support such as the designs proposed in this study. The crowns with a $0.5 \mathrm{~mm}$ buccal porcelain thickness, supported by wave-like projections in the zirconia core, showed significant improvement in fracture resistance compared to the crowns with the same thickness but without the addition of wave-like projections. This might be explained by the zirconia waves, which may prevent shear forces from developing along the buccal porcelain-zirconia interface. If that is the case, shear stress is interrupted by the wave projections, which act as support along the buccal interface. However, further studies, eg, using finite element analysis are needed to analyze the different developing stresses of this design to confirm this explanation.

Improvement in fracture resistance was also seen when the buccal porcelain was protected by a cap-like projection protecting the porcelain from high occlusal loads. These semimonolithic designs, however, are more complicated for the dental technician to prepare, and additional time is thus needed for the CAD-up compared to monolithic and traditional bi-layered designs where default settings can be used.

When comparing translucent zirconia with high-translucent zirconia materials in the present study, it was seen that the design was able to compensate for unfavorable mechanical properties of the weaker high-translucent material to some extent, by using a microcoating or wave design instead of one of the other designs investigated. In the present study, two zirconia materials were used each with a different degree of translucency. The first represents one of the early generations of translucent zirconia, and the second represents a more recent one with improved optical properties. There are different ways to gain increased translucency in densely sintered zirconia. One way is to alter the grain sizes and indirectly the proportional distribution of grain boundaries. ${ }^{8,31}$ Some producers also use materials with a higher content of cubic crystals at the expense of the tetragonal ones. ${ }^{13}$ The toughness of zirconia is one of the properties that make the material durable compared to all other dental ceramics. ${ }^{32,33}$ When a crack is formed during loading and stress concentrations develop at a critical flaw or in the crack-tip area in the material, phase transformations occur that close the crack tip, leaving it in residual compression, which prevents propagation of the crack, so-called transformation toughening. ${ }^{32,33}$
This advantageous toughening property depends on both grain size and what crystal phases are present in the material. Hence, for the transformation toughening mechanism to be effective, both grain size and phase types present must be balanced. Larger grains tend to transform spontaneously, and grains that are too small do not transform at all, leading in both cases to a less tough material. In both cases, strength and toughness will decrease proportionately in the material. ${ }^{27}$ If the traditional, optically dense Y-TZP type of zirconia is considered to represent the type of zirconia with optimal composition and grain size, it might be expected that strength and toughness decrease the greater the changes from this ultimate composition. If this is the case, it might explain the results of the present study where the material with the higher translucency showed lower fracture resistance regardless of crown design.

Some studies, however, do not show substantial differences between translucent zirconia and traditional Y-TZP in respect of fracture resistance. ${ }^{9}$ Such results may imply that different brands of translucent zirconia perform differently compared to each other, probably because translucency is gained by using a variety of compositions and production methods, which results in different properties. This leads to contradictory results and makes it difficult to compare different studies as they were testing different brands of translucent zirconia.

Analysis of the failure type of the crowns shows two modes of fracture among the tested designs. The crowns that were microcoated with a $0.3 \mathrm{~mm}$ veneer showed the same fracture pattern as the monolithic crowns (complete fractures). Since the porcelain layer was thin and the supporting core material was relatively thick, the porcelain was probably subjected to mainly compressive forces during loading, which in general, ceramics are very resistant to compared to shear and bending forces. ${ }^{27}$ Consequently, the core material became the limiting factor, resulting in complete fractures. Almost the same result was seen in the cap design where the buccal veneer was protected by the occlusal cap. This crown design was close to the monolithic design, which was probably the reason for a similar fracture pattern.

Cohesive veneer fractures, on the other hand, were more common in designs with a $0.5 \mathrm{~mm}$ buccal veneer with a wave-protected configuration but only with the translucent and not with the high translucent material. The reason for this might be that the tensile stress building up in the core material exceeds the materials' load-bearing capacity before or at the same time as this limit is reached in the porcelain. The stronger translucent material resists the load that exceeds 
the porcelain capacity, resulting in a cohesive fracture in the porcelain only. This is the same fracture behavior as detected in clinical and laboratory studies on fully veneered Y-TZP restorations with a comparable thickness of veneer layers. ${ }^{3,4,6}$ Thick layers of porcelain upon zirconia, regardless of coping thickness or design, therefore appear to be more liable to cohesive veneer fracture due to the low fracture strength of the porcelain material.

In vitro studies have shown that the modulus of elasticity of the supporting die material has an influence on the fracture outcomes of tested materials. ${ }^{25,34}$ It is recommended that the mechanical properties of dies are close to those of the dentin, ranging from 10 to $15 \mathrm{GP}$, to prevent them from fracturing during loading tests and to prevent the generation of irrelevant fracture data of the crowns. In the present study, however, the die material used has a modulus of elasticity slightly lower than the recommended range. Nevertheless, all the dies remained intact during all the artificial aging steps and load to fracture tests. Fracture loads were also comparable to previous laboratory studies using dies with elastic properties within the desired range. ${ }^{6,9,25}$ In addition, the die preparation design and the resin cement used in this study were standardized for all the groups tested and were used according to the manufacturers' instructions.

One of the limitations of laboratory studies is the difficulty to choose which aging procedures would produce comparable clinical results. Previous studies have shown the significance of combining the mechanical tests with water and thermocycles to produce the fatigue effect of zirconia. ${ }^{22-25}$ Other studies performed accelerated aging protocol in autoclave for aging zirconia using high temperatures reaching $134^{\circ} \mathrm{C}$, which could not be considered representative for the oral environment. ${ }^{35,36}$ The specific aging protocol of the present study was performed in attempt to mimic the real clinical environment that the crowns would be subjected to it and to make comparisons with previous studies with similar methodologies. ${ }^{6,9,24,25}$

There are always limitations in relation to the methodology of laboratory studies, and the results of the present study may not fully represent the clinical situation. The oral environment is far more complex and is affected by multiple factors that it is impossible to fully mimic, and it is difficult to extrapolate results from the mechanical loading test alone. One such limitation in the present study is the fact that the steel contact counterpart in the load to fracture apparatus has a different modulus of elasticity than the clinical contact counterpart (food, teeth), which influences on the stress magnitude suffered by the material. Therefore, the results of the present study need to be confirmed by results from clinical studies.

\section{Conclusion}

Within the limitations of the study, the following can be concluded:

- Translucent and high-translucent zirconia crowns might be used in combination with $0.3 \mathrm{~mm}$ microcoating porcelain layer with semimonolithic design to enhance the esthetic properties of restorations without significantly decreasing the fracture resistance of the crown.

- If $0.5 \mathrm{~mm}$ porcelain layer is needed for a semimonolithic crown, wave design or cap design might be used to increase the fracture resistance.

In both cases, the fracture resistance gained is likely to be clinically sufficient as the registered fracture loads were high in relation to expected loads under clinical use. Clinical studies are, however, needed to confirm those findings.

\section{Acknowledgments}

The authors would like to thank Umm Al-Qura University for funding. The Panavia F 2.0 cement material was kindly provided by RH Dental ApS, Gentofte, Denmark. A draft of the abstract was presented at the CED-IADR/NOF oral health research congress, September 23, 2017, in Vienna, Austria, and at the Annual Meeting of Academy of Dental Materials, October 6, 2017, in Nuremberg, Germany. This article has never been previously published.

\section{Author contributions}

FB and PVvS contributed to study conception, design, data acquisition, analysis, and interpretation and drafted and critically revised the article. PS, EP, and CL contributed to study conception and design and drafted and critically revised the article. All authors gave final approval of the version of the article to be published.

\section{Disclosure}

The authors report no conflicts of interest in this work.

\section{References}

1. Rimondini L, Cerroni L, Carrassi A, Torricelli P. Bacterial colonization of zirconia ceramic surfaces: an in vitro and in vivo study. Int J Oral Maxillofac Implants. 2002;17(6):793-798.

2. Denry I, Kelly JR. State of the art of zirconia for dental applications Dent Mater. 2008;24(3):299-307.

3. Le M, Papia E, Larsson C. The clinical success of tooth- and implantsupported zirconia-based fixed dental prostheses. A systematic review. J Oral Rehabil. 2015;42(6):467-480. 
4. Sailer I, Makarov NA, Thoma DS, Zwahlen M, Pjetursson BE. Allceramic or metal-ceramic tooth-supported fixed dental prostheses (FDPs)? A systematic review of the survival and complication rates. Part I: single crowns (SCs). Dent Mater. 2015;31(6):603-623.

5. Raigrodski AJ, Yu A, Chiche GJ, Hochstedler JL, Mancl LA, Mohamed SE. Clinical efficacy of veneered zirconium dioxide-based posterior partial fixed dental prostheses: five-year results. J Prosthet Dent. 2012;108(4):214-222.

6. Larsson C, El Madhoun S, Wennerberg A, Vult von Steyern P. Fracture strength of yttria-stabilized tetragonal zirconia polycrystals crowns with different design: an in vitro study. Clin Oral Implants Res. 2012;23(7):820-826.

7. Conrad HJ, Seong WJ, Pesun IJ. Current ceramic materials and systems with clinical recommendations: a systematic review. J Prosthet Dent. 2007;98(5):389-404.

8. Zhang Y. Making yttria-stabilized tetragonal zirconia translucent. Dent Mater. 2014;30(10):1195-1203.

9. Johansson C, Kmet G, Rivera J, Larsson C, Vult Von Steyern P. Fracture strength of monolithic all-ceramic crowns made of high translucent yttrium oxide-stabilized zirconium dioxide compared to porcelainveneered crowns and lithium disilicate crowns. Acta Odontol Scand. 2014;72(2):145-153.

10. Nakamura K, Harada A, Inagaki R, et al. Fracture resistance of monolithic zirconia molar crowns with reduced thickness. Acta Odontol Scand. 2015;73(8):602-608.

11. Worni A, Katsoulis J, Kolgeci L, Worni M, Mericske-Stern R. Monolithic zirconia reconstructions supported by teeth and implants: 1 - to 3-year results of a case series. Quintessence Int. 2017;48(6):459-467.

12. Abdulmajeed AA, Lim KG, Narhi TO, Cooper LF. Complete-arch implant-supported monolithic zirconia fixed dental prostheses: a systematic review. J Prosthet Dent. 2016;115(6):672-677.

13. Zhang F, Inokoshi M, Batuk M, et al. Strength, toughness and aging stability of highly-translucent Y-TZP ceramics for dental restorations. Dent Mater. 2016;32(12):327-337.

14. Munoz EM, Longhini D, Antonio SG, Adabo GL. The effects of mechanical and hydrothermal aging on microstructure and biaxial flexural strength of an anterior and a posterior monolithic zirconia. J Dent. 2017;63(1):94-102.

15. Venezia P, Torsello F, Cavalcanti R, D'Amato S. Retrospective analysis of 26 complete-arch implant-supported monolithic zirconia prostheses with feldspathic porcelain veneering limited to the facial surface. J Prosthet Dent. 2015;114(4):506-512.

16. Bomicke W, Rammelsberg P, Stober T, Schmitter M. Short-term prospective clinical evaluation of monolithic and partially veneered zirconia single crowns. J Esthet Restor Dent. 2017;29(1):22-30.

17. Yoshinari M, Derand T. Fracture strength of all-ceramic crowns. Int $J$ Prosthodont. 1994;7(4):329-338.

18. Vult von Steyern P, Ebbesson S, Holmgren J, Haag P, Nilner K. Fracture strength of two oxide ceramic crown systems after cyclic pre-loading and thermocycling. J Oral Rehabil. 2006;33(9):682-689.

19. Luthy H, Loeffel O, Hammerle CH. Effect of thermocycling on bond strength of luting cements to zirconia ceramic. Dent Mater. 2006;22(2):195-200.
20. Shahin R, Kern M. Effect of air-abrasion on the retention of zirconia ceramic crowns luted with different cements before and after artificial aging. Dent Mater. 2010;26(9):922-928.

21. Kelly JR. Clinically relevant approach to failure testing of all-ceramic restorations. J Prosthet Dent. 1999;81(6):652-661.

22. Kohorst P, Dittmer MP, Borchers L, Stiesch-Scholz M. Influence of cyclic fatigue in water on the load-bearing capacity of dental bridges made of zirconia. Acta Biomater. 2008;4(5):1440-1447.

23. Anusavice KJ, Kakar K, Ferree N. Which mechanical and physical testing methods are relevant for predicting the clinical performance of ceramic-based dental prostheses? Clin Oral Implants Res. 2007;18(3):218-231

24. Bakitian F, Seweryniak P, Papia E, Larsson C, Vult von Steyern P. Fracture strength of veneered translucent zirconium dioxide crowns with different porcelain thicknesses. Acta Biomater Odontol Scand. 2017;3(1):74-83

25. Mahmood DJ, Linderoth EH, Vult Von Steyern P. The influence of support properties and complexity on fracture strength and fracture mode of all-ceramic fixed dental prostheses. Acta Odontol Scand. 2011;69(4):229-237.

26. Whitworth JM, Walls AW, Wassell RW. Crowns and extra-coronal restorations: endodontic considerations: the pulp, the root-treated tooth and the crown. Br Dent J. 2002;192(6):315-320,323-327.

27. Richerson DW. Modern Ceramic Engineering: Properties, Processing and Use in Design. 3rd ed. Boca Raton: CRC Press; 2006.

28. Swain MV. Unstable cracking (chipping) of veneering porcelain on all-ceramic dental crowns and fixed partial dentures. Acta Biomater. 2009;5(5):1668-1677.

29. Lassila V, Holmlund I, Koivumaa KK. Bite force and its correlations in different denture types. Acta Odontol Scand. 1985;43(3):127-132.

30. Gibbs CH, Mahan PE, Mauderli A, Lundeen HC, Walsh EK. Limits of human bite strength. J Prosthet Dent. 1986;56(2):226-229.

31. Kim MJ, Ahn JS, Kim JH, Kim HY, Kim WC. Effects of the sintering conditions of dental zirconia ceramics on the grain size and translucency. $J$ Adv Prosthodont. 2013;5(2):161-166.

32. Maerten A, Zaslansky P, Mochales C, et al. Characterizing the transformation near indents and cracks in clinically used dental yttria-stabilized zirconium oxide constructs. Dent Mater. 2013;29(2):241-251.

33. Hannink RHJ, Kelly PM, Muddle BC. Transformation toughening in zirconia-containing ceramics. J Am Ceram Soc. 2000;83(3):461-487.

34. Yucel MT, Yondem I, Aykent F, Eraslan O. Influence of the supporting die structures on the fracture strength of all-ceramic materials. Clin Oral Investig. 2012;16(4):1105-1110.

35. Pereira GKR, Muller C, Wandscher VF, Rippe MP, Kleverlaan CJ, Valandro LF. Comparison of different low-temperature aging protocols: its effects on the mechanical behavior of Y-TZP ceramics. J Mech Behav Biomed Mater. 2016;60:324-330.

36. Cotes C, Arata A, Melo RM, Bottino MA, Machado JP, Souza RO. Effects of aging procedures on the topographic surface, structural stability, and mechanical strength of a $\mathrm{ZrO} 2$-based dental ceramic. Dent Mater. 2014;30(12):396-404.
Clinical, Cosmetic and Investigational Dentistry

\section{Publish your work in this journal}

Clinical, Cosmetic and Investigational Dentistry is an international, peer-reviewed, open access, online journal focusing on the latest clinical and experimental research in dentistry with specific emphasis on cosmetic interventions. Innovative developments in dental materials, techniques and devices that improve outcomes and patient satisfac-
Dovepress

tion and preference will be highlighted. The manuscript management system is completely online and includes a very quick and fair peerreview system, which is all easy to use. Visit http://www.dovepress. com/testimonials.php to read real quotes from published authors. 